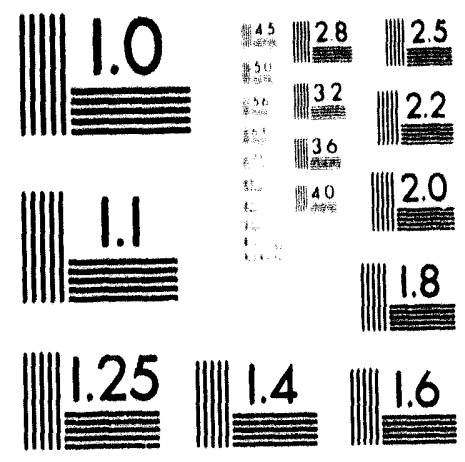



SANDO3-0032

Unlimited Rolease

Printed August 1993
Distribution

('ategory UC-515

\title{
Methodology for Testing Metal Detectors Using Variables Test Data
}

\author{
Debra D. Spencer \\ Special Projects Department \\ and Dale W. Murray \\ Entry Control/System Engineering Department
}

\begin{abstract}
By extracting and analyzing measurement (variables) data fron portal metal detectors whenever possible instead of the more typical "alarm"/"no-alarm" (attributes or binomial) data, we can be more informed about metal detector health with fewer tests. This testing methodology is an alternative to the typical binomial testing and in many ways is far superior.
\end{abstract}




\section{Table of contents}

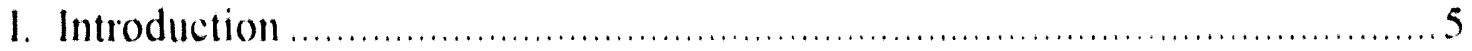

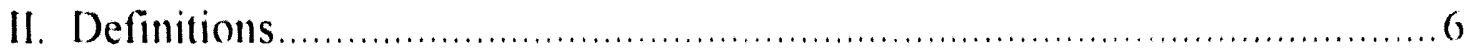

Figure 1 Typical Alarm Threshold Interval..........................................6

Figure 2 Orientations ................................................................. 9

III. Why Use Variables Data ........................................................ 10

Figure 3 Variables Data ................................................................. 11

IV. Obtaining Variables Data From a Metal Detector................................ 11

V. Types of Tests in a Variables Data Sampling Scheme for Metal Detector

Testing .............................................................................. 16

VI. Detector Characterization Testing ......................................... 17

VII. Detector Initialization ............................................................. 20

Figure 4 SPC Charts ..................................................................... 23

VIII. Periodic Reevaluation ...................................................... 24

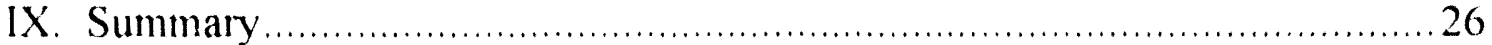

XI. Reference Sources ............................................................ 27

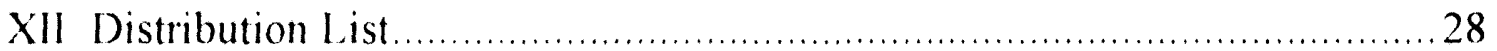




\section{Methodology for Testing Metal Detectors l sing V'ariables Test Data}

\section{Introduction}

The intent of this paper is lo document an alternative testing methodology for metal detectors across the D()l: complex and to briefly describe an associated laptop computerbased system for obtaining and analyzing the test data. This methodology is an alternative to that in the Metal Detector Guide of April 1991. By extracting and analyzing measurement (variables) data whenever possible instead of the more typical "alarm"/"noalarm" (attributes or binomial) data. we can be more informed about metal detector health with fewer lests.

Past testing of metal detectors and similar devices has relied upon evaluation of attributes ("yes"/no" or binomial) data. where the extent of information in each test is limited to knowing whether or not an alarm was generated. The limited amount of information in such data drives the number of tests neeessary to meet regulatory requirements to unacceptably high levels.

Clearly. the detector itself operates at a more quantitative level of information prior to making the internal logical decision whether or not to alarm. The detection of metal in a metal detector is really the detection of eddy currents. Fddy currents are electrical currents that result when a conductive material such as metal is exposed to a time-varying magnetic field. The magnitude and phase relative to the energizing ficld are determined by the electromagnetic and geometrical characteristics of the target. Secondary magnetic fields are generated by the eddy currents themselves and are detected by the metal detector's receiver coils. The detection signal is processed and compared to an internal threshold value and used with other signal characteristics (such as rise time and duration of the signal) to make an alarm decision. This internal detection signal is variables data and if access to this data is available then a great deal more information can be obtained.

Since regulatory requirements are typically stated in terms of confidence levels, which are based upon attributes data, regulatory agencies such as the Department of Energy and the Nuclear Regulatory Commission may eventually want to revise their stated requirements if the use of variables data for metal derector testing becomes more widespread.

This paper examines the use of variables data in addition to attributes data in the testing and evaluation of metal detectors. Section II reviews relevant definitions and terminology. Section III examines in more detail why we want to use variables data. Section IV considers a method to easily obtain variables data. Section V details the types of tests needed for a metal detector evaluation program that uses variables data. Sections VI through VIII give the specifics of the various types of tests. Section IX summarizes this report, and Section $X$ provides references. 


\section{Definitions}

The following definitions and terminology are used throughout this document.

Alarm threshold imerial - a smail interval of values of the primary metal detector alarm variable that result in alarm variability. If one uses detector test data and plots probability of detection (POD) versus the observed response variable (peak voltage, for instance), the result is a sharp-kneed curve that has a constant POD value of zero rising to a constant $P O D$ value of one. The interval of response values involved in the knee of the curve is the alarm threshold interval. Values of the primary response variable that exceed this interval consistently result in alarms. Values below this interval consistently result in no-alarms. Values within the interval sometimes result in alarms and sometimes in no-alarms. For completeness, we will use the phrase "consistently result in alarms" to mean that 20 out of 20 passes all result in an alarm.

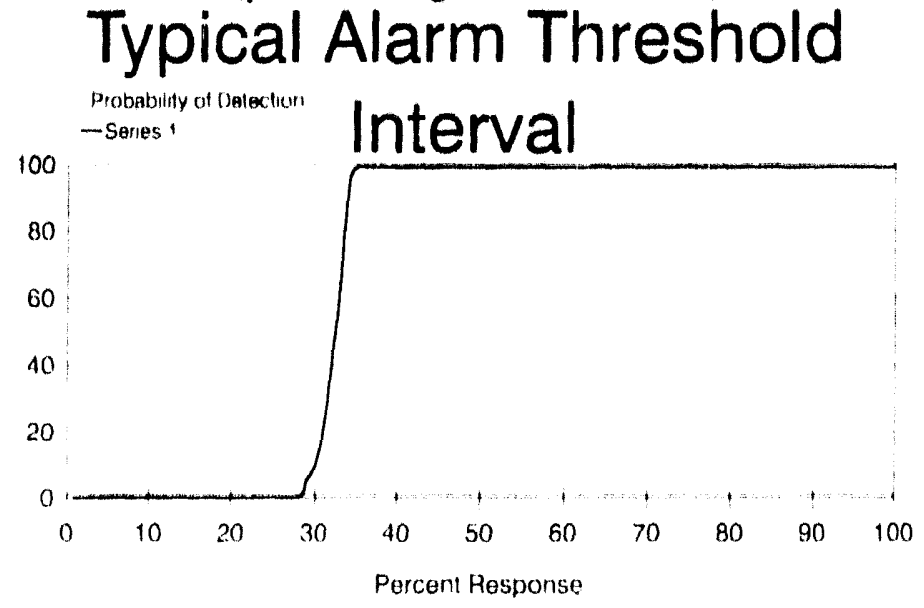

Figure 1

Aperture - the opening in the metal detector bounded by the floor, the top cross member, and the side panels.

Attributes data - data of the pass-fail variety. Attribute performance indicators are usually based upon counts of the pass-fail data, such as number of tests of the metal detector where the test object was indeed detected.

Daily (or shifi) minimal function test - a go/no-go test performed frequently to determine if the detector will respond to a conspicuous quantity of metal (such as a guard's service revolver or pistol). This test is generally a single trial and is not intended to meet any statistically based requirements. This report does not attempt to describe or define how the daily or shift minimal function test is conducted.

Detector characterization test - a test performed immediately following initial installation, after any movement of the metal detector, and after any rework activities. Its purpose is to establish the metal detector's response to the appropriate program setting in terms of:

- Worst-case test object,

- Worst-case orientation of test object,

- Worst-case location of test object, 
- Worst-case detection direction for metal detectors used bidirectionally,

- Worst-case combination of all of the above parameters,

- Maximum amount of extrancous metal that can be tolerated, and

- Effects of body mass, etc.

Detector zone - one of three metal detector zones that evenly divide the archway of the metal detector vertically. The zones are referred to as ankle. waist, and head.

Diagnostic-analysis and corrective-action state - the state of determining what the problem is and how to correct it, which is entered upon test failure in either the initialization test or the periodic reevaluation. Frequently, detector characterization testing (at least in an abbreviated form) will be necessary to return from this state to normal operation.

DOE: weapon test objects - objects defined by the Depariment of Energy to be the threat against metal detectors used for contraband detection at entry control portals located at DOE facilities. These normally will be among the objects used in field testing of metal detectors used for entry control.

DOE SNM-shiclding test objects - the object or objects defined by the Department of Energy or the specific facility to be the threat against metal detectors used for exit control at that facility. These will normally be among the objects used in field testing of metal detectors that are used for exit control.

Initialization test (in-plant performance test) - a metal detector test that has three purposes: 1) to determine appropriate sensitivity settings that ensure that the operation of a metal detector meets DOE requirements, 2) to determine the alarm threshold interval, and 3) to initialize mechanical testing values to be used during periodic reevaluation. This test should be performed 1) after any detector characterization test, 2) after any maintenance or repairs to the detector, and 3 ) after any operational change to the detector's sensitivity settings. These test procedures should be consistent with the facility's actual operational procedures.

Mechanical testing - testing that uses mechanical devices such as inclined planes instead of test walkers to transport test objects through the detector.

Periodic reevaluation (functional operation test) - a test intended to verify the continued normal detector operation following initialization test. A periodic reevaluation should be conducted at intervals that average once weekly. These test procedures use a mechanical test device and statistical process control techniques to evaluate whether metal detector operation has changed. 
Range (of a set of dara) - the difference between the maximum value and the minimum value of a set of datia

Realiste kesteng - testing that involves test walkers carrying D()E test objects through the detector in a manner consistent with mutine operational procedures.

S'onsurity mapping - plotting the relative sensitivity to a specific test object at regularly distributed points within the detector aperture. These points lie on a vertical plane normal to the direction of travel through the detector.

Standard sensitivity wnit - approximately $1 / 100$ of the full sensitivity range of a metal detector. Various metal detectors have sensitivity scales that are graduated in different increments. Some detectors have scales incremented in tenths, some in hundredths, and others in thousandihs. In order to standardize the scales for the purposes of this report, a standard sensitivity unit is defined as approximately $1 / 100$ of the full range of sensitivity. For instance, a metal detector has sensitivity settings from 0 to 999; one standard sensitivity unit would be equivalent to 10 of that detector's sensitivity units. If a detector has sensitivity settings from 1 to 99 , it is sufficient to definc one standard sensitivity unit for this detector as one sensitivity setting.

Statistical process control (SH'(') - the use of statistical techniques to monitor the steps in a process. SPC began with the development in the 1920s of Shewhart control charts to measure and respond to the variability in a process. SPC is an aid in the discovery of all types of problems and in identifying the type and degree of corrective action required. While we are making use of these techniques for evaluating the process of metal detector operation over time, they are more commonly used as a quality tool to control manufacturing processes.

Test configuration - the combination to be used in a series of tests of either 1) test object, test walker, detector zone, test-object/body location, and test-object orientation for realistic testing, or 2) spherical test object, mechanical track, and mechanical track position in the metal detector aperture for mechanical testing.

Test object - the choice of object to be used in a particular series of metal detector tests. For realistic testing, the test object will be from the set of DOE weapons test objects and DOE SNM-shielding test objects. In the case of mechanical testing, the test object will usually be a standard spherical test object.

Test-object hody location - the location on the test-walker body where the test object is to be carried during realistic testing. For example, the location could be 1) the inside of the left ankle, two inches above the floor, or perhaps 2) the back of the waist in the middle. 


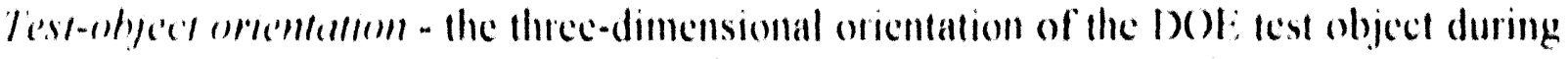
realistic testing. (See figure 2 for typical weapon test object orientations.)

\section{ORIENTATIONS}
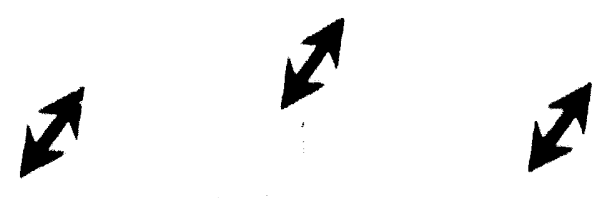

Figure 2

Test records - the recorded results of 1) detector characterizations,

2) initialization lesting, and 3) periodic recvaluations

Test walker - the person who, during a realistic test, walks through the metal detector carrying the test object to determine how the detector is operating. The test walker should be between four feet eight inches and six feet live inches tall. The test walker's mass is usually unimportant; however, test walkers should be evaluated during detector characterization testing to

determine that their mass does not significantly affect the performance of the detector. If mass has a significant effect, test walkers of the worst-case general mass should perform subsequent testing. No metal other than the test targets should be carried by the test walker. A careful evaluation of each test valker should be made prior to performing any tests. A sensitive hand-held metal detector is helpful in making this evaluation. Also, before beginning the lests, the test walker shall make one or more passes through the detector without a test object to verify that the detector shows little or no response to the presence of the individual.

Test-walker varich hility - variation in test data caused by differences in 1) testers, 2) walk style, and 3 ) speed. Test walkers should undergo a period of training to learn how to reduce data variability. Extraneous motion such as nodding the head, swinging the arms, or swinging the hips should be held to a minimum. During "head" tests, test walkers should focus on an eye-level spot on the wall in front of them to keep their heads from nodding. Velocity of the walk shall be a normal pace of approximately 30 inches per second as determined by a slow count (one pace per second). Velocity can range between 21 inches per second and 42 inches per second and still be acceptable. During an "ankle" test, the pace of the walker shall be normal with foot placement such that the ankle that carries the test target shall consistently swing through the detector aperture or pause within the aperture. depending on which is worst-case as determined by the detector characterization. If normal facility operational procedure dictates that persons being screened must pause within the aperture, the test walker shall pause in the detector aperture. Variability caused by placement of the test object can be reduced through standard procedures and the use of aids such as ankle holsters. 
Variahles data - real-number-valued data that are usually physical measurements, such as size, volume, temperature, or voltage.

Worst-case(s) - the one or more combinations of test objects, detector zones, testobject/body locations, and orientations that are the hardest to detect. Whichever combination is more difficult to detect than all others will be considered the ahsolute worst-case.

\section{Why Use Variables Data}

Testing using attributes data to evaluate a metal detector requires much morc testing than using a test scheme based upon both attributes and variables data if regulatory requirements are to be met.

Using the DOE metal detector testing requirement of 85 probability of detection at a .95 confidence level, a single-phase testing strategy, using attributes data and allowing no missed alarms, requires 19 identically performed tests. A testing strategy allowing one miss (i.e., zero or one miss results in a successful test; two or more missed alarms result in test failure, which leads to investigation, possible rework, and repeat of detector initiation tests) requires 30 identically done tests. A similar testing strategy allowing up to two misses requires 40 identically done tests. Various multiple-phase sampling schemes can also be devised that meet the $.85 / .95$ requirement. Clearly, if one is to test several test objects at several locations against the $.85 / .95$ requirements, the quantity of tests quickly gets too large to be practical.

By using variables data in addition to attributes data, we can examine the value of the peak response parameter, which is typically the primary alarm variable. From this we can make inferences about probability of detection with many fewer tests. A .95 confidence interval around the estimate for worst-case passage of the threat object can be established. As long as this confidence interval is above the alarm threshold interval, the detector is working in an acceptable manner. In addition, direct comparisons can be made more easily between different test objects, different positions and orientations, and many other factors. Detector variability can be assessed, and no-alarm conditions can be investigated in a more informed manner. 
Consider the three example cases described in figure 3 . I et us assume that five consistent metal detector tests are performed and result in four alarms and one missed alarm. Our best estimate of probability of detecion. based solely upon attributes data, is 80 with a 95 confidence interval for the probatility of detection of $(.38,995)$. ('learly we learned little from the test beyond the fact that the detector alarms at least part of the time. On the other hand, if the variable of peak voltage is being measured, consider three hypothetical cases that resulted in Figure 3. Prior experimentation with this detector showed that the alarm threshold interval was very close to 8 volts, and the detector became saturated at 15 volts. All three different cases in Figure 3 could easily have each resulted in four out of five alarms, just as in the attributes case. However, the three cases now have very different interpretations due to the variables, data. Case I shows a metal detector operating consistently but a little too close to the alarm threshold interval; the sensitivity setting should be turned up a little. Case 2 shows a detector consistenlly operating well above the threshold with one quite different test value; we need to determine what caused it and what we can do to prevent it during routine operation.

Case 3 shows a detector operating in an inconsistent, and therefore unacceptable, manner; rework, movement, replacement, or some other change is needed.

Another method of analysis, statistical process control (SPC), will be introduced later in this document for use with variables test data. While SPC can be used successfully with attributes data, doing so requires an unacceptably high number of tests. In general, the number

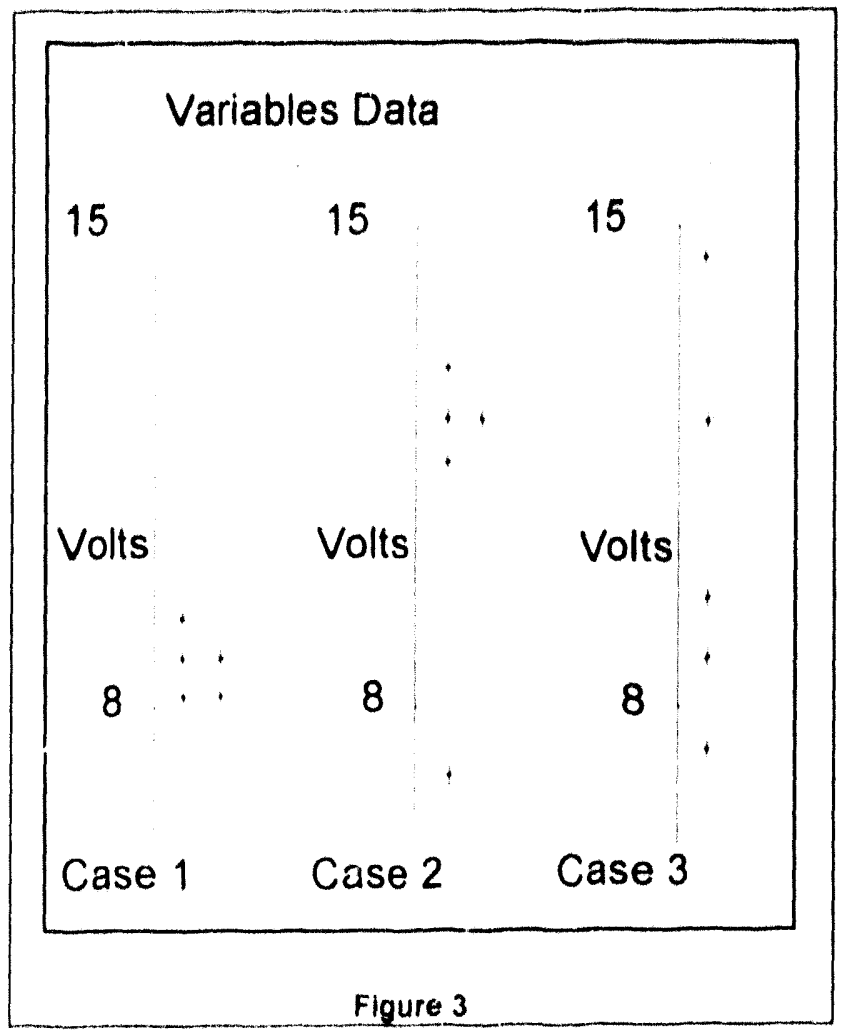
of trials should be large enough to ensure several failures per test if using SPC to examine attributes data.

\section{Obtaining Variables Data From a Metal Detector}

\section{Methods for Obtaining Data}

Currently, no metal detector is designed with an output port for the explicit purpose of providing variables data. Most metal detectors do, however, provide a visual display that indicates the detector's response to stimuli. The display is usually a series of ten to twelve light emitting diodes (LED's) that form a bar meter. The number of illuminated LED's 


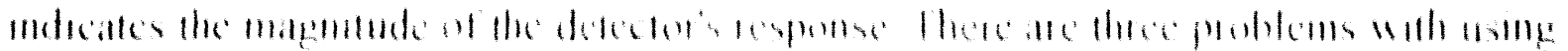



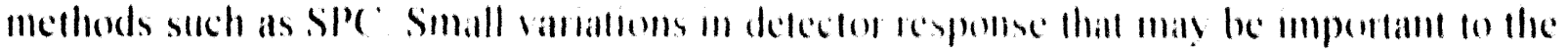
lesting method can be "lost" because of the puantized nature of this data. The second problem is that the meter does not always reflect the full scale of the metal detector's response. An example of this is the case of the le (ide ( $i$ strophysics $A$ T This defector has an L.ED meler that displays only the lower lifty to sive percent of the detector's response. The third problem is that even if the display has sufficient resolution. the display ean be difficult to read accurately "on-the-fly." An example of this is the case of the fiederal

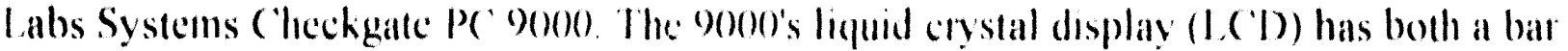
meter and a numerical readout of the esponse. but. unless the detector alarms, the display fluctuates so rapidly that accurate reading of the meter is nearly impossible. Fontumatcly. a port that allows the tester to access the raw data intended for the visual display is often associated with the display

Most metal detectors seem amenable to the extraction of varbables data; however, the methods for obtaining the data vary, and interfaces for the most common types will need to be included in any test kit to be based upon variables testing. Three examples of metal detectors that have been lested suceessfully at Sandia National I aboratories using variables data are the Infinclics series 500 , the E(i\&e $;$ Astroplyssics AT, and the ()utokumpu Metor 120.

The Infinetics series 500 has, as an option, an I.CD voltmeter display. The delector's peak response for each target passage is recorded and displayed. The response is measured in millivolts and has more than sufficient resolution for variables testing. (ne drawback to this system is that the data must be recorded manually.

The EG\&G Astrophysics AT has a connector located on the back of the electronics unit with an output signal that drives the $1,1: D$ ) bar meter. This analog signal is located on pin 24 of the auxiliary connector. The signal is typically 0 to 15 volts in magnitude with the alarm threshold set al around 8 volts. These levels vary slightly from detector to detector The tenth and final LED is lit at the point that the detector alams. The data can be taken manually using a digital storage oscilloscope or recorded antomatically by a computer equipped with an analog-to-digital interface card. This computer interface has been accomplished at SNL, in the Metal Detector Automated Test Track located in the metal detector test laboratory. This interface is somewhat awkward and may prove inappropriate for field lesting. Care must be taken when using this analog output not to connect the test cable to the ground pin on the comnector. Reference ground must be accomplished by ensuring that the oscilloscope is plugged into the same grounded power circuit as the detector. If the ground reference is taken from the ground pin on the DB-25 connector, the test cable forms a conductive loop that, if moved slightly, causes a large interference signal in the metal detector. 




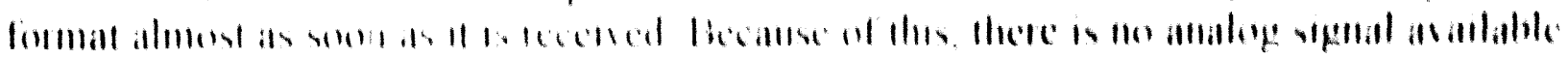

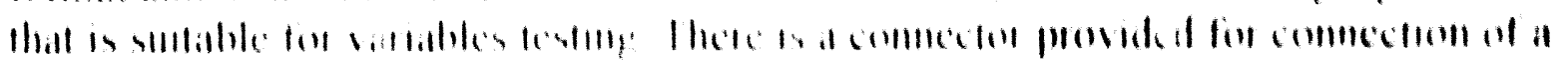

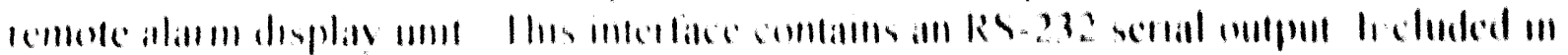

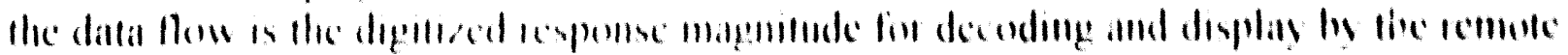

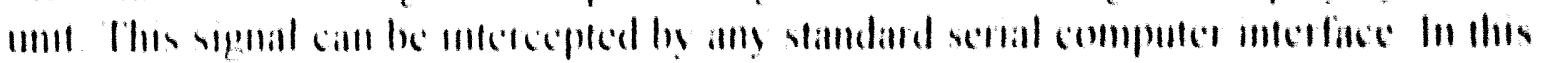

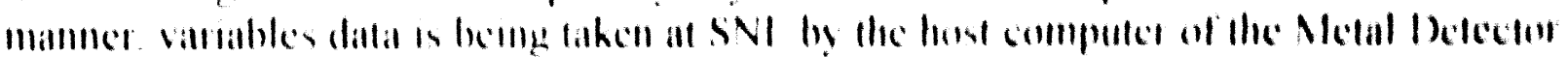

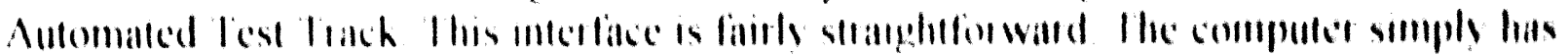

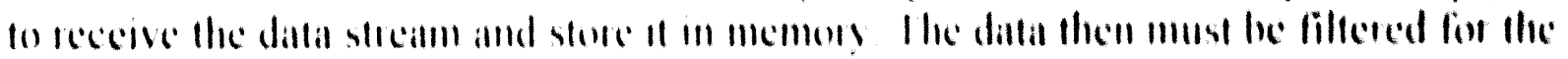



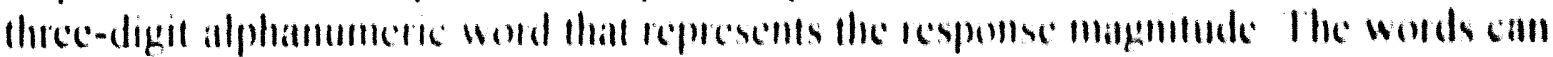

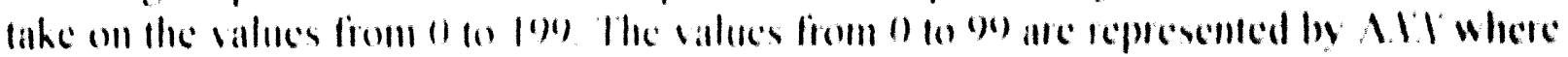

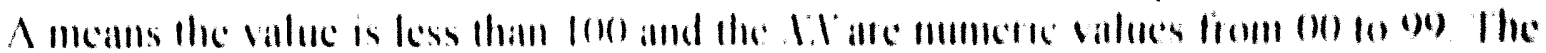

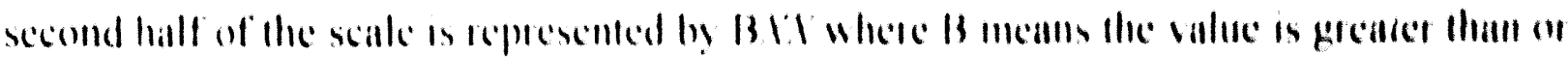

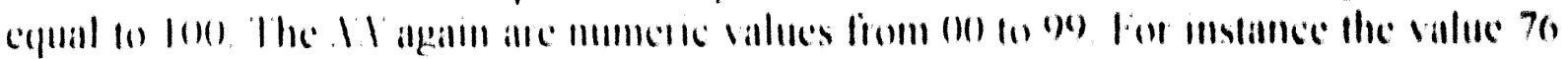
would be represented by 176 while the value 159 would be replescented by 1354 the alarm threshold is 1300 , or exactly half of hle scalle

\section{( ollecting und Analyaing Datu}

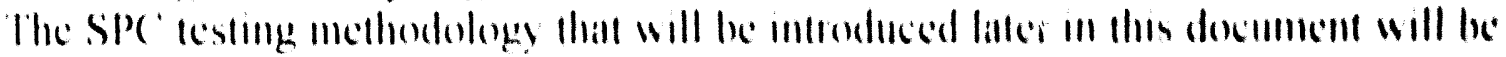

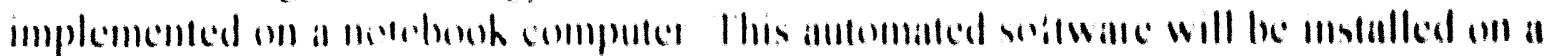



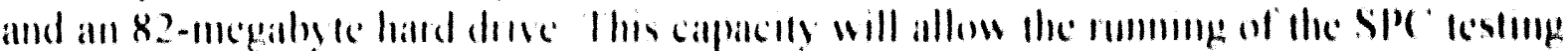

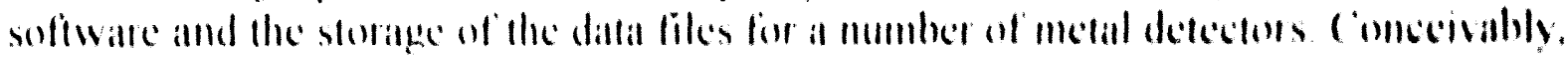
all metal detector testimg! all any simgle litciliey combl be accommmodated on a single colilpulet

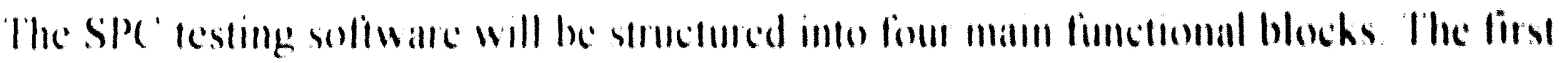

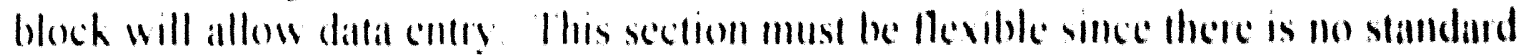


will be included for some detectors, he dettal entry will necessinily he manmal while

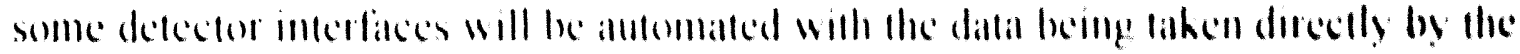
compulter

The second functional hock of the soltwanc will be the dala reduction and analysis section. Various graphs and statistical analyses will be generaled, depending upon the lest type. The code will perform the necessaly statistical calculations, as an example, the results of the SPC data analysis will be plofted on the SPe chant and alamms penentated if the delection process is found to be out of combol bounds 
The third solliware block will imerpret results and provide advice. The statistically based ndvice will involve decisions such as whether the detector should be adjusted or whether the enter should take a walt-and-sec posture

The fourth and finnl software bloch will output reports. This section will provide the decumentanon of the esting procedures and provide evidence that the facility is or is not in compliance with performance refuirements.

\section{Mechanicul Teating}

Varmous lest chotecs must be made in establishing a metal detector test program. One is

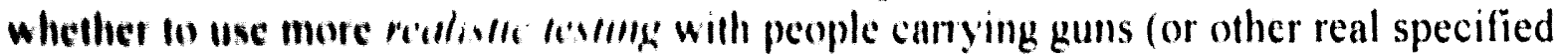
flieat objects) through the metal detecter in realistic ways An alternative to this type of

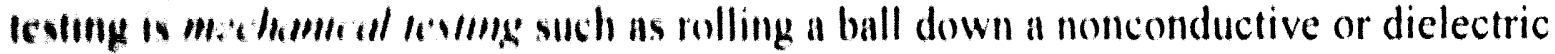
uncline postluned llurough the metal detector aperture; mechanical testing generally has

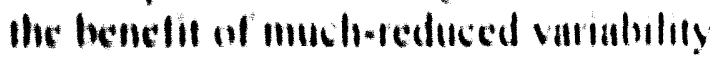

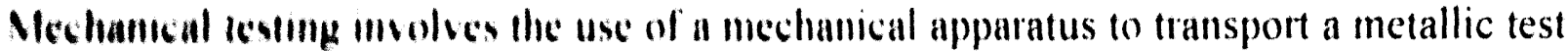
whect thouph the metal detector. The test object may be realistic, such as a handgun, or may be a yplerical or cylindrical est object. The main reason for using a mechanical test apparntus for metal detector lestlung is to reduce the variability and increase the repeatibility of the datn lo further reduce the variability of the detector response, a viliencal test olyect is best A geometrically complex object like a handgun will produce a wide sarely of revponse magnitudes whe'l passed through the detector in various

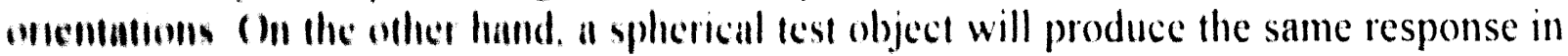
a metal detcentr repandless of the oncentation. A realistic test object can be used stcecovfully fin low variability testlng if great care is takell to ensure that the object



The Metal Detector Automaled Test Track operated in the Sandia metal detector latwormon is a computer-controlled mechanical test track, which has shown that mechamical testing can produce very low variability data. However, this test apparatus is nen portuble and therefore not uscfiul for field testing.

Seceral appronches for mechamical testing can be used in the field. The simplest is to allach the test object to a nonconductive pole so that the test object can be passed through the detector by someone grasping the end of the pole. This method is useful for some mappung procedures but probably will not reduce the variability of the data sufficiently for SPF I wo remaining sources for variability remain. It is still difficult to pass the test ohject llowough the detector in exactly the same place each time. and the speed is difficult Io control All chllancement to this method is to use a nonconductive guide or track to assist in controlling the placement of the test object. $A$ guide that has been used with pood results at Sandia is a section of tiberglass I-beam. In this case the I-beam is 
supported at either end with tripods, and a plastic pipe is used to push or pull the test object down the guide through the detector. With careful measurements of the guide's position(s) through the detector, the guide can be placed in exactly the same position(s) for the next round of tests. The variability of the data taken with this method depends on the detector's sensitivity to object speed.

In an effort to reduce the variability of the test-object speed, Sandia is developing a gravity-feed field test track. The gravity-feed field test track operates on the idea that a metallic sphere will roll down an inclined plane with highly repeatable velocity. If each time the inclined track is positioned in the detector it is carefully placed in exactly the same location with the same inclination, the response of the metal detector to the same metallic sphere rolling down that track will be highly repeatable. A gravity-feed field test track that has an eight-foot length with a rise of six inches will produce a test-object velocity of about three feet per second at the mid-point of the track. To use this apparatus, one would place the track through the detector with the mid-point of the track positioned halfway through the detector. The lateral and height placement is not critical on the first test and can be chosen somewhat arbitrarily. The exact lateral and height placement of the track must be recorded for future testing. The track is then ready for use. The spherical test object is released from the top end of the track without pushing. The ball will roll down the track, and the detector response is recorded. For subsequent tests the track must be located in exactly the same position for the data to have a low variance.

Additional data can be achieved by carefully choosing the placement of the track or choosing a sphere size and material that will cause alarms. Not only can the magnitude of the response be obtained but it can be verified that the alarm circuitry is operating correctly. If the detector does not alarm at the first effort, the track can be placed closer to one of the side panels. Also a larger sphere or a material that is detected more easily can be used. Most metal detectors have a stronger response to carbon steel than to non ferromagnetic metals such as aluminum. Be careful not to use a stainless steel because stainless alloys of steel have a much higher resistivity than common steel, and some alloys of stainless steel are not magnetic.

The track will be constructed of low-weight materials to make the track highly portable and non-metallic materials to prevent interference with the metal detector's performance. Another design characteristic is that the setup of the apparatus will be simple, fast, and highly repeatable. Any system that is difficult to set up would not likely be located with the precision that may be required for SPC testing. The combination of the light-weight, gravity-feed, field test track and the notebook data acquisition/analysis computer shoulc provide a powerful, easy-to-use system. 


\section{Types of Tests in a Variables Data Sampling Scheme for Metal Detector Testing}

The distinction among types of testing needs to be clear in the minds of the testers. Types of interest in this document include 1) detector characterization testing, 2) detector initialization or acceptance testing, and 3) periodic reevaluation of the detector. During detector characterization testing (which follows initial detector installation, any movement of the detector, program change, or any rework), the detector is tested to determine worst-case combinations of threat object, location, and orientation. Defector initialization is performed after any detector characterization testing or when the metal detector sensitivity settings are changed. Its primary purpose is to adjust the sensitivity settings for proper operation. Periodic recvaluation is a more abbreviated testing aimed at periodically reestablishing that operational behavior has not been altered since the last initialization testing. In addition, daily or shifi minimal fincthon festing is performed by metal detector operators with an object known to create an alarm, such as a service revolver, to establish that the detector is capable of alarming. Other types of testing beyond the scope of this document include 1) quality process control testing done by the manufacturer as the metal detectors are produced and 2) laboratory testing done by manufacturers in the process of developing new designs or by facilities such as Sandia National Laboratories in the context of technology R\&D. In-plam metal detector 'valuation programs should include characterization, imtialization, and periodic reevahation, in addition to daily or shifi minimal function kesting hy operators.

The use of variables data will allow us to learn more about the operation of the metal detector using less data; however, a need for consistency between trials becomes even more obvious when using variables data, although the same need exists whether using attributes or variables data. During any group of trials that will be considered identical in statistical analysis, variability must be kept to a minimum. Sources of possible variability in metal detector test data include:

- changes in metal detector location,

- test walker (walking style, speed, height, metallic implants, other),

- test-object path through the metal detector,

- test-object orientation,

- changes in test objects,

- external electromagnetic interference, and

- others.

The testing methodology presented here will use hoth realistic kesting and mechancal testing in an effort to remain realistic while simultaneous/y reducing variahility. Test walkers will be used only for detector characterization and initialization testing. Mechanical testing will be used during initialization and periodic reevaluation. The use of 
both test walkers and mechanical testing in initialization serves the purpose of informal correlation of the two types of testing. Thus much of the variability can be removed with the use of mechanical testing for periodic reevaluation, but the mechanical test results will have been initialized at a time when realistic testing results proved acceptable for meeting regulatory requirements.

\section{Detector Characterization Testing}

The purpose of detector characterization testing is to determine worst-case combinations of threat object, location, and orientation for a particular metal detector. In addition, responses to test-walker characteristics are studied and operational sensitivity settings are approximated. At the time of test, the metal detector has already been installed in a fixed location, and a program number, if available, has already been chosen.

Sensitivity mapping both characterizes the sensitivity within a metal detector's walkway and locates the point(s) of lowest sensitivity. Finding the point(s) of lowest sensitivity is essential to proper sensitivity adjustment and to subsequent performance testing. There are three basic components to any mapping procedure.

1) There is the method of carrying the test object through the detector.

2) There is the method of taking data and how it is analyzed.

3) There is the way that test points are selected and how they are used to zero in on areas of low sensitivity.

Prior to designing a mapping test procedure the person designing the test must weight the strengths and weaknesses of each factor and choose the combination of components that best suits his particular needs.

The methods of carrying the test object through the detector are divided into two broad categories, test walkers and mechanical means. The main advantage to using a test walker to carry the test objects through the detector is that no apparatus other than the test object is required to perform the test. Another advantage of using a real person to carry the test object through the detector is that the data collected in this manner is more realistic and, therefore, more suitable for determining the appropriate sensitivity setting for operation. The disadvantages to using a walk tester are the variability that a test walker introduces into the results, and the requirement that care must be taken to insure that the walker is clean. The main advantage to using a mechanical means to transport the test object through the detector is that the results of tests performed with mechanical means can be highly repeatable. The disadvantages of mechanical testing are the need for additional apparatus, and loss of portability. Also, data taken with a mechanical method should not 
be used to determine the operational sensitivity setting. The minimum setting that is determined through the use of mechanical testing is often too low for consistently detecting threat items on a person.

It is a simple thing to have a person carry a test object through a detector, however, there are several things that must be done to ensure that the results of the test are of any value. First, the person that is to carry the object through the detector has to be screened to locate any metallic items on the person of the walk tester. Common items that must be removed are eye glasses, belt buckles, jewelry, calculators, and any other metallic items that may interfere with the test. Another thing to consider is that the person to perform the test should not have any physical characteristic that prevents them from passing the test item through any of the test points. For instance, a person that is too short cannot carry the test object through the upper locations in the detector aperture. A person when walking with a normal gait will cause the test target to vary in orientation, velocity and placement. These variations can cause significant variations in the data. Making an effort to walk in a consistent manner can reduce this variability, but may not reduce it enough for certain applications.

Depending on the design, the use of mechanical apparatus to carry the test target through the detector can greatly reduce variation in the results. The design of the apparatus can be as simple as a nonconductive hand-held rod to pass the target through the detector to a computer controlled motorized test track. While the hand-held rod will likely introduces less variation than a walk tester, it cannot match the high repeatability of a motorized test track. Unfortunately, motorized test tracks are not usually portable enough for field applications. However, designs of non-motorized test tracks can be light and portable enough for field use and can provide results that are comparable to the motorized tracks.

Other aspects of performing a sensitivity map are the data variable and the ways in which the data are taken. For the first method, the object is passed through the detector aperture, and the sensitivity is adjusted to find the minimum setting required to reliably detect the object. Thus, the data variable in this method is the alarm threshold and the units are sensitivity points. This method assumes that the response-to-sensitivity relationship is linear. A weakness of this approach is that some metal detectors have analog sensitivity control knobs instead of digital controls. For detectors that have this analog type of control, determining the sensitivity level accurately is very difficult.

A second method of taking data is possible when there is an interface that allows response measurements to be made. A sensitivity setting is selected that produces a strong response to the object but does not saturate the detector at any location. The test object is then passed repeatedly through the detector at each test location, and an average response is recorded for that location. In this method, the alarm/no-alarm response of the metal detector is probably of little interest. For this method, the data variable is detector 
response (at a given sensitivity) and the units may vary, but are usually normalized to percent response. The main weakness of this method is that not all detectors have a variables data port.

There are basically two approaches to choosing test points and locating the point(s) of lowest sensitivity when sensitivity mapping metal detectors. The first approach is to use a rectangular grid to divide the metal detector walkway into cells. The second approach is to perform a vertical cut through the center of the metal detector, then based on the information derived from the vertical cut. perform a horizontal cut(s) across the metal detector at the point(s) of lowest vertical sensitivity.

The strength of the grid approach is that the entire walkway is sampled at regularly spaced test points, and there is a higher probability of detecting any anomalous sensitivity locations. If any locations within the walkway have unusually high or low sensitivities, there is a good chance of finding that location with the grid technique. Because regularly spaced locations throughout the entire walkway is mapped in this procedure, the grid approach is most suited for laboratory characterizations of metal detectors where the performance characteristics of a detector are of prime importance.

The major drawback to the grid approach is that, due to the size of walk-through metal detectors, the grid cannot provide high resolution without the number of cells becoming prohibitively high. A typical metal detector has a walkway width of thirty-two inches and a height of seventy-two inches. A thirty-six-cell grid that is four wide by nine high has a cell center spacing of eight inches. This wide spacing of the test points may not be of sufficient resolution for some applications.

The crosscut method of mapping makes the assumption that horizontal crosscuts form a family of sensitivity curves having varying magnitudes but similar general shapes .

Laboratory tests indicate this to be a good assumption. The purpose of the initial vertical cut is to find the vertical location that $i$; the most likely location of the weakest, horizontal curve. Once the vertical locetion(s) of lowest sensitivity is found, the horizontal crosscut can be mapped in high resolution.

The advantage of the crosscut method is that an area of low sensitivity can be mapped with high resolution with a small number of passes. The weaknesses to the crosscut approach are that less information about the general performance characteristics is obtained and this method is less likely to detect unusual areas of sensitivity or insensitivity.

Another way of reducing the number of test points without reducing the resolution relies on the fact that the sensitivity for all current metal detectors is considerably higher near their side panels where their coils are located than it is at the center of the walkway. Once 
this has been proven for the metal detector under testing, the areas near the panels can be ignored.

A metal detector of the same dimensions as the previous example can be mapped using the crosscut technique by first making a vertical cut with sixteen test points. Then an eight-test-point horizontal crosscut is made through the center half of the walkway (ignoring the outer one-quarter of the width on each side). This mapping has a total of only twenty-four test points with much higher resolution in the indicated area of interest. Vertical spacing of the test points has been reduced to four and a half inches with :1 horizontal spacing of only two inches.

Test objects used in device characterization always include realistic test objects such as weapons and can include spherical test objects. However, realistic test objects, carried by several test walkers, should be used to determine worst-case test object, orientation, and location when determining the correct operational sensitivity setting. Also because tests have shown that the area of lowest sensitivity for a symmetrical test object is not always the area of lowest sensitivity for an actual threat object, it is advisable to use actual threat items for sensitivity mapping.

No matter which method is used for sensitivity mapping, it is important to perform enough passes at each test position. If response measurements are being taken, the tester should record and average several repetitions at each: position. If sensitivity adjustments are being made, enough passes are required to meet whatever definition of "reliably detect the object" is applicable.

The laptop computer will be used during this phase of the testing to record, summarize, and plot test data across test walkers, test objects, sensitivity settings, locations, and orientations, to draw a map of metal detector response, and to print a report for documentation purposes. The tester wil' be reguired to input certain test parameters. Tentative recommendations for operational sensitivity settings will be generated.

\section{Detector Initialization}

The purpose of detector initialization is 1) to determine the appropriate sensitivity setting for the installed detector to meet regulatory requirements, 2) to establish the detector alarm threshold interval, and 3) to prepare for future periodic testing. Detector initialization is performed after any detector characterization and any time the operational sensitivity settings are to be changed.

Detector initialization testing starts with realistic testing using test walkers and concludes with mechanical testing aimed at setting a baseline for comparison during periodic reevaluation. All test results will be collected, summarized, and displayed by the laptop. 
The worst-case test(s) was determined during detector characterization. For all tests, the test walker shall pass the target through the center of the detector aperture unless detector characterization indicates that worst-case locations are further than two inches from the center, in which case the test walker should strive to move the test object through the detector aperture as close as possible to the worst-case location. The test walker should pause within the aperture only if that is nomal facility operational procedure.

If any metal detector is used for both entry and exit weapons screening, testing should be conducted in the worst-case detection direction as determined during detector characterization testing. If a detector is used for both entry weapons screening and exit SNM-shielding screening, the detector must be tested against both types of test objects in the appropriate directions.

It is assumed that prior to conducting this test, sufficient work has been done to determine both the approximate sensitivity setting and the program number (if applicable) for a particular metal detector to achieve proper detection of the defined threat objects.

Each of the test combinations determined during detector characterization testing will be tested, with more testing for the absolute worst-case. For each test combination, test both below and above the required sensitivity setting, teminating in the following two-step acceptance test at slightly above the alarm threshold interval. The laptop will track all test results.

To accept the sensitivity setting for operational use, perform the following two-step test.

STEP 1. For the absolute worst-case combination, the test walker shall make 20 consistent passes through the metal detector, with the sensitivity set such that the peak voltage or other primary alarm variable is producing values sufficiently above the alarm threshold interval, with the test walker trying to do exactly the same thing each time. Failure to detect on any pass results in failure of the test. Failing the test usually means that the sensitivity is not high enough. When operating at very high sensitivity levels, it could mean that a diagnostic-analysis and corrective-action state needs to be entered, perhaps with assistance from the metal detector manufacturer, to determine necessary changes in the metal detector environment, program, or some other factor prior to completion of initialization testing.

STEP 2. Next, for each of the other test combinations, the test walker should make five consistent passes. Failure to detect on any pass results in test failure and initiation of a diagnostic-analysis and corrective-action state. 
All these test results, including alarm/no-alarm information, are collected by the laptop. The alarm threshold interval is determined to be the interval of all values between the minimum test value causing an alarm and the maximum test value not causing an alarm.

Computer output from this phase of the testing will consist of a recommended sensitivity setting and the value of the alarm threshold interval. These values will be calculated using the recorded results of the test. Other output options are empirical probability distribution plots for the alarm threshold interval and plots showing test results across objects, locations, and sensitivity settings. These should be filed in the facility's test records.

At this point, fix the sensitivity level for the remainder of the test and for continued operation afterward. Any later operational readjustment requires a repetition of initialization testing. The recommended minimum sensitivity setting will be slightly above the minimum sensitivity during testing that had no corresponding no-alarms.

Next, a mechanical test track, described in a preceding section, will be used to initialize the process for weekly periodic reevaluation.

The primary analysis methodology used with regard to mechanical testing will be the SPC chart. The laptop software will aid facility testers by generating these automatically. SPC charts will allow weekly testing to be kept at minimal levels. The SPC chart in Figure 4 shows data taken in weekly subgroups of size 5 using the ankle location. The data shown represent 30 weeks of testing with a metal detector performing at acceptable levels.

SPC charts plot weekly averages of peak voltage as well as the range of the values. The process (in our case, the process of metal detector operation over time) is considered to be unchanged (in control) as long as the weekly additions stay within the $3 \sigma$ control limits plotted on the control charts. Other trends, such as extended runs above the average, are also noted since they could lead to interesting questions about patterns in weekly response.

Making all tests as identical as possible is important for using the SPC charts. There is a great need to reduce the variation introduced by the testing process itself. Control charts use within weekly subgroup variation to put limits on how much variation should exist between the weekly subgroups. Not only should testing within a weekly subgroup be kept to a minimum; it is even more important to minimize variation between weekly subgroups. 
The use of SPC charts during initialization has two purposes. One is to determine process capability; the other is to initialize the control charts and determine the values to be used as $3 \sigma$ control limits. Experts generally agree that testing subgroups of size 5 is acceptable. A minimum of 50 trials is recommended to determine process capability, which includes

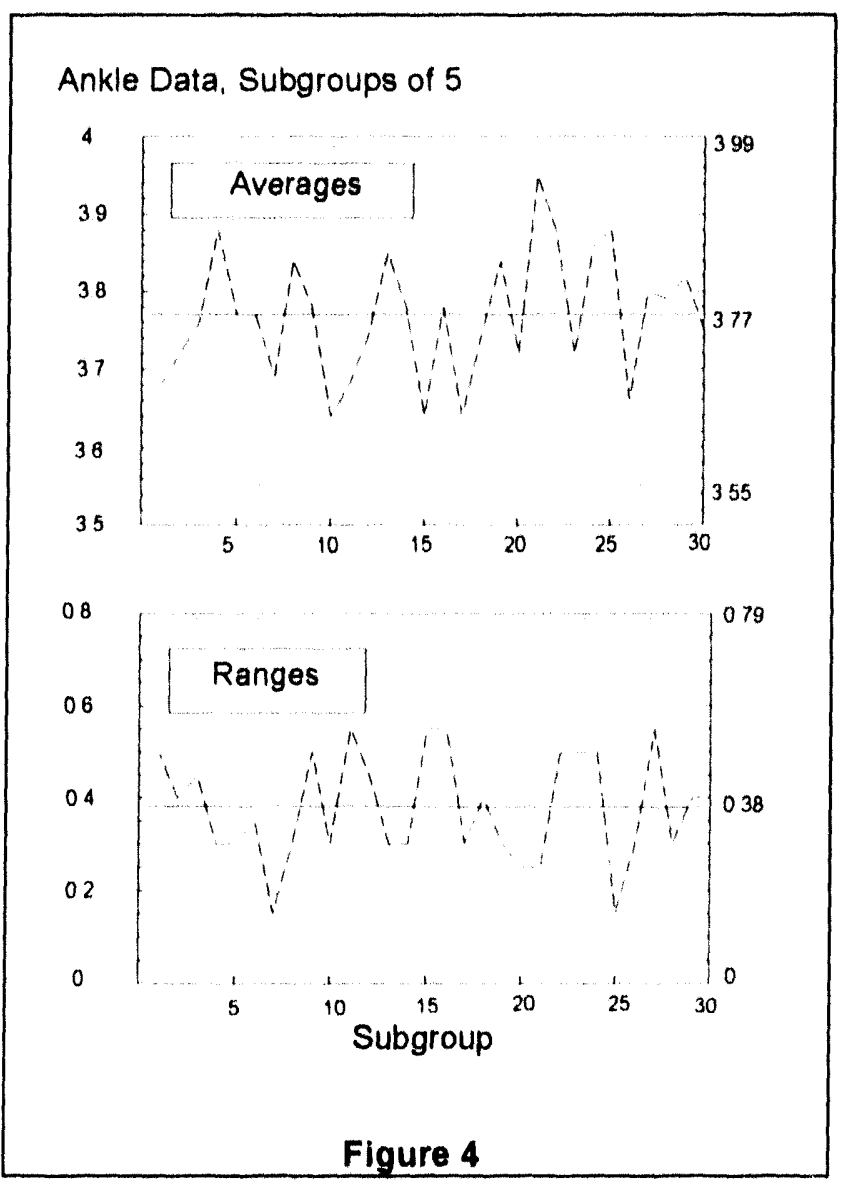

1) calculating control limits and 2) determining if any initial data indicate a process that is stable, in control, and capable of being tracked with SPC. Whether or not the process values lie within product specifications or, in this case, meet regulatory requirements has already been established via realistic testing. To fully initialize the control charts, you will need approximately 25 subgroups of five tests each to minimize the impact of any extreme values. Since doing 125 is not usually convenient in one setting, and since the process may have some natural time variation, the initialization can take place over the first few weeks. Fifty trials can be done initially, followed by 5 weeks of fifteen trials each. At the end of this time, the process capability study is updated, and initialization is considered complete if the results are satisfactory. During the 5-week study period, any values outside the trial control limits should lead to a repetition of the realistic two-step acceptance test

described above. Afterward, periodic reevaluation (next section) will track metal detector operation.

For the SPC initialization and continuing periodic reevaluation, testing one point in roughly the center of the detector is sufficient since all transmitter coils have some effect on center operation. In this manner, the test track height can be fixed, reducing variability from test setup. Floor positioning can be made repeatable by permanently marking the floor.

The control limits are plotted using the following formulas. For the Averages Chart, the upper and lower control limits are given respectively by the formulas

$$
U C L=\overline{\bar{X}}+0.577 \bar{R} \text { and } \quad L C L=\overline{\bar{X}}-0.577 \bar{R}
$$


where $\overline{\bar{X}} \bar{X}$ is the sum of all sample means divided by the number of samples and $\bar{R}$ is the sum of all sample ranges divided by the number of samples. The control limits for the Ranges Chart are

$$
C Y=2.114 \bar{R} \text { and } L C Y=0
$$

An estimate of the standard deviation is

$$
\sigma=\bar{R} / 2.33 \text {. }
$$

A more accurate estimate of the standard deviation for use in estimating $\pm 3 \sigma$ population limits is

$$
\sigma=\sqrt{\frac{n \sum x_{1}^{2}-\left(\sum x_{1}\right)^{2}}{n(n-1)}}
$$

where the $x_{j}$ 's are the test values and $n$ is the total number of values.

In certain cases, using a median chart based upon the middle data value instead of the average will make more sense. A determination of which is more appropriate will be made using the lap-top software and used thereafter in periodic reevaluation.

If the process does not seem capable of being in control, resort to realistic testing but continue to collect variables data in addition to the attributes data and use the laptop to generate charts. Experts may be able to help you analyze your data and improve your process so that it can be controlled via SPC charts.

The laptop will generate the following reports and values during initialization testing: - plots of test-walker data, - alarm threshold interval,

- recommended sensitivity setting, - results of process capability analysis, and - SPC charts with initialization data plotted.

\section{Periodic Reevaluation}

After initialization testing is completed, weekly periodic reevaluation will test samples of size 5 using the same mechanical testing procedure used at the end of detector initialization. The laptop computer will continue to be used to record results, plot a new SPC chart including the latest data, and give advice about the need for further evaluation at that time based upon the new data. The only outputs from routine periodic reevaluation are new SPC charts. 
By observing values on the control charts, one has an indication that the process of metal detector operation has changed. Test group averages and ranges that fall outside the $\pm 3 \sigma$ are indications that one should go into a short diagnostic state to determine if there is a problem that needs fixing. Observations of any changes in environment or procedure and a repeat of the realistic acceptance test are sufficient reaction. If the detector fails the realistic acceptance test, a diagnostic-analysis and corrective-action state becomes necessary.

In addition to values falling outside the control limits on the Averages or Ranges Charts, one should observe and react to the following non random events:

- cyclic behavior.

- bimodal behavior,

- runs, where several suecessive values are on the same side of the mean, and - trends.

For normally distributed data, an interval of $t .3 \sigma$ about the mean contains $99.7 \%$ of all the values. Thus one could expect that approximately 3 out of 1000 lests would result in values falling outside the control limits, even if no change has occurred. As a result, oceasionally $S P C$ charts will indivate a problem when none exists.

When the process really has changed, the $S P C$ charts will need to be reinitialized. This does not always mean that the sensitivity settings of the detector will need to be changed. For instance, the testers may have become more consistent with their test methods.

Reinitialize the SPC charts when:

- the process changes (new subgroups do not fit in with old ones),

- initial limits were trial ones, or

- there is some indication of inflated limits such as 1) the presence of range values beyond UCL or 2 ) the average range exceeds $2 / 3$ of range values. In this case we can use median ranges instead of average ranges 


\section{Summary}

In summary, SPC methods should work for many metal detector installations. Even if they do not, the use of variables data instead of attributes data should give more basic information about the operation. In addition, these concepts show promise for expansion to other security-related devices. 


\section{Reference Sources}

Roger W. Berger, Thomas H. Hart. Shatisical Process ('omerol, A (iunde for

A. V. Feigenbaum. Toral (Luahuy ('omrrol, 3rd ed. New York: MeGran-Hill, 1983


1972


ASQC-Quality Press, 199?

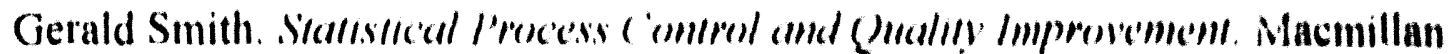
Publishing Company, 1991

Donald J. Wheeler and David S. Chambers. IInderstumding Sil' : 2nd ed. SPC Press. Inc., 1992 


\section{DISTRIBUTION}

Distribution Services

Omer of Scientific and Technical

Information

I. $\mathrm{S} \mathrm{DOL:OOR}$

PO BOC 62

(Gak Ridge. TN 378.31

Imtemal Distribution:

8523.2 (entral Technical Files

7141 Technical Library (5)

7151 Technical Publications

7613.2 Document Processing for DOE:OSTI (10)

323 Robert Lasterling

95(0) Dennis Miyoshi

9502 L I ibrary (10)

9904 Ron W. Moya

9531 D. J (iangle

9531 D D. Spencer (5)

9538 Steve Ortiz.

9545 I ( i Waddoups

9945 B II Gardner

9545 M K Snell

9548 J F C Chapek

9548 F.J. Conrad

9548 B. T. Kenna

9548 D. W. Murray (10)

9548 J R. Rodriguez

9549 B. J. Steele

9549) I. D. Miller

9561 J D. Williams

9561 H. D. Arlowe 

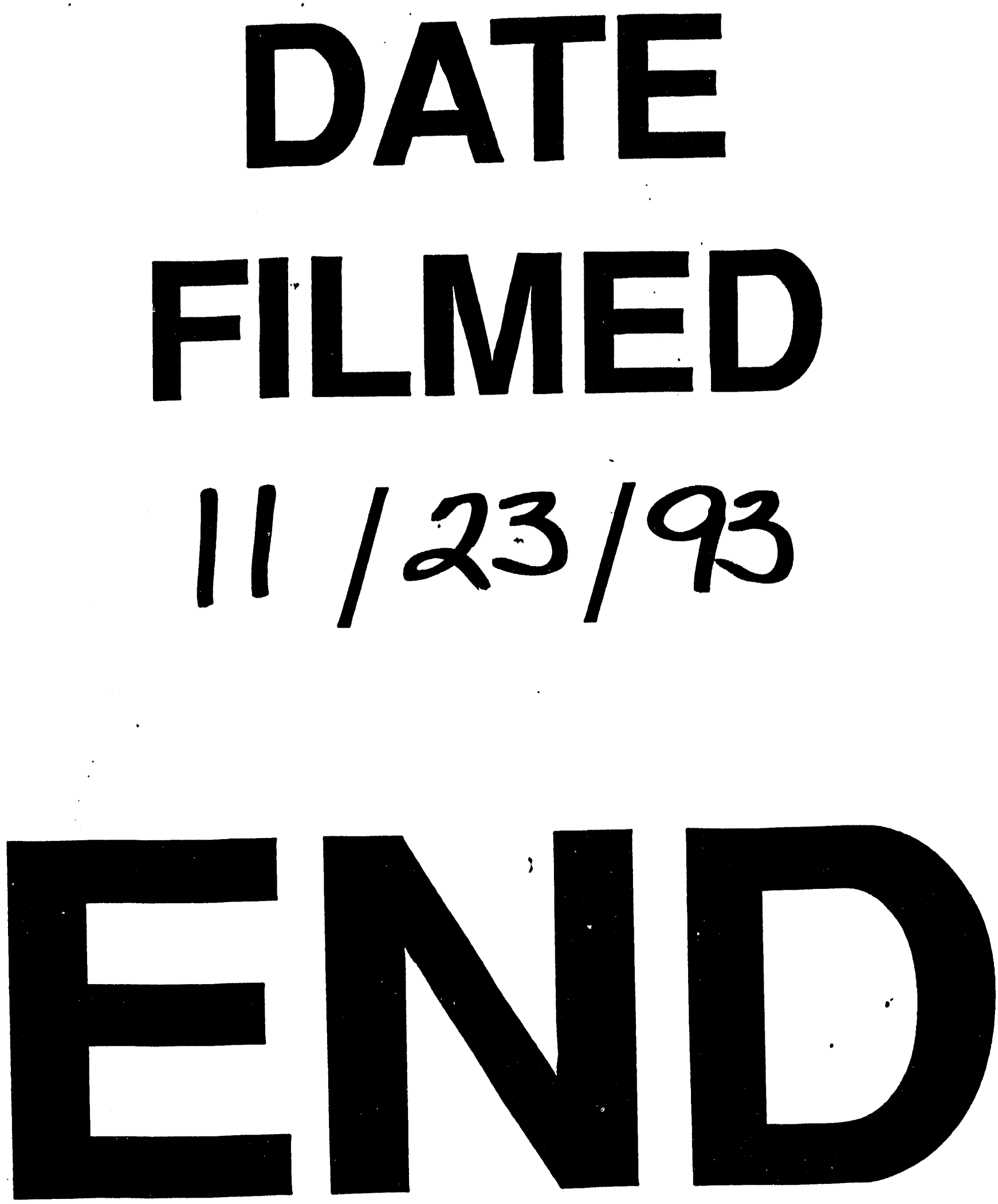


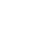

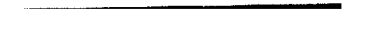

